\title{
Dielectric and Magnetic Nonlinear Materials for NLTLs
}

\author{
Elizete G. L. Rangel ${ }^{1}$, José O. Rossi ${ }^{1}$, Joaquim J. Barroso ${ }^{2}$, L. P. Silva Neto ${ }^{3}$ and E. Schamiloglu ${ }^{4}$ \\ National Institute for Space Research (INPE), São José dos Campos, Brazil \\ ${ }^{2}$ Technological Institute of Aeronautics (ITA), São José dos Campos, Brazil \\ ${ }^{3}$ Federal University of São Paulo, São José dos Campos, Brazil \\ ${ }^{4}$ University of New Mexico, Albuquerque, USA
}

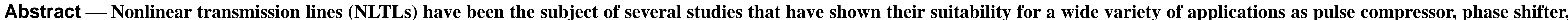

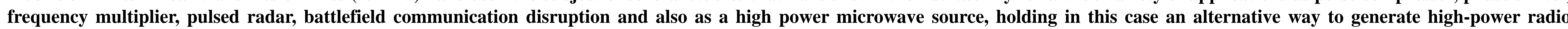

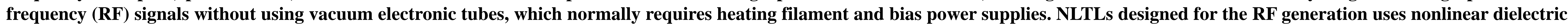

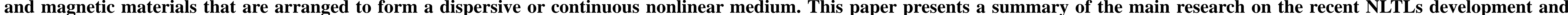

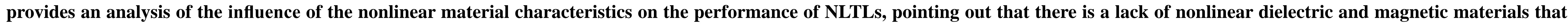

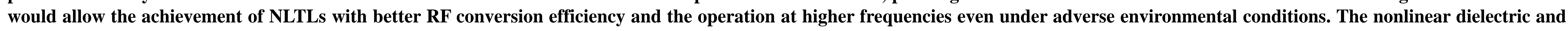
magnetic materials for NLTLs applications need to have characteristics such as highly nonlinear behavior, low losses, and thermal stability.

\section{Types of NLTLs}

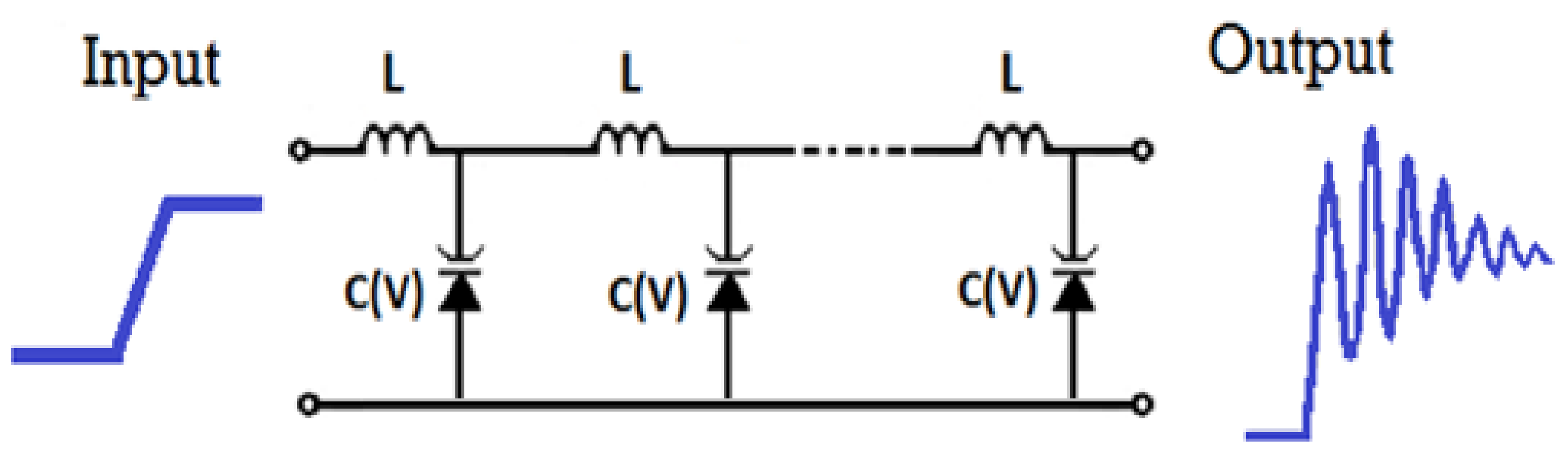

Fig. 1. Capacitive NLTL.

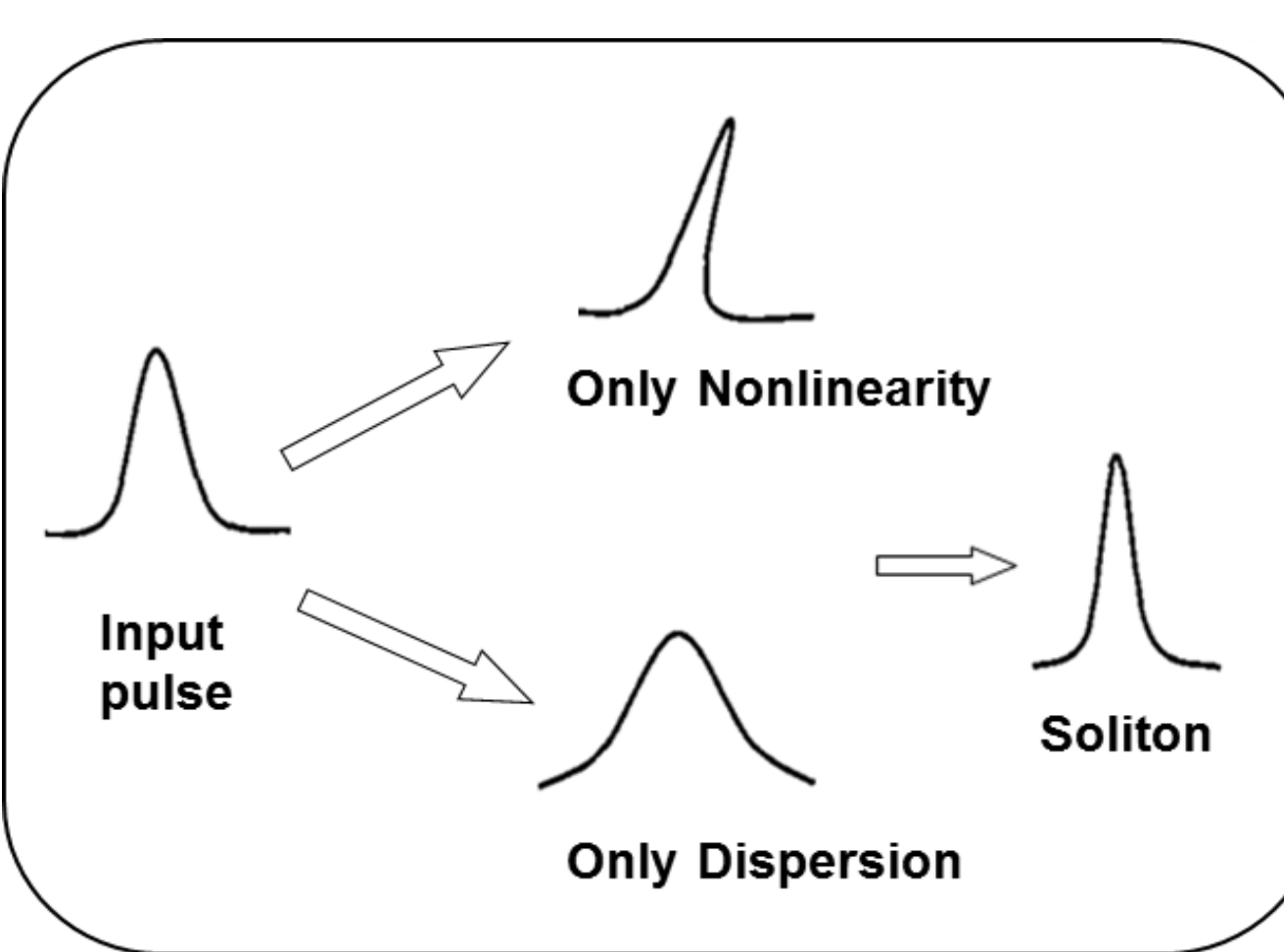

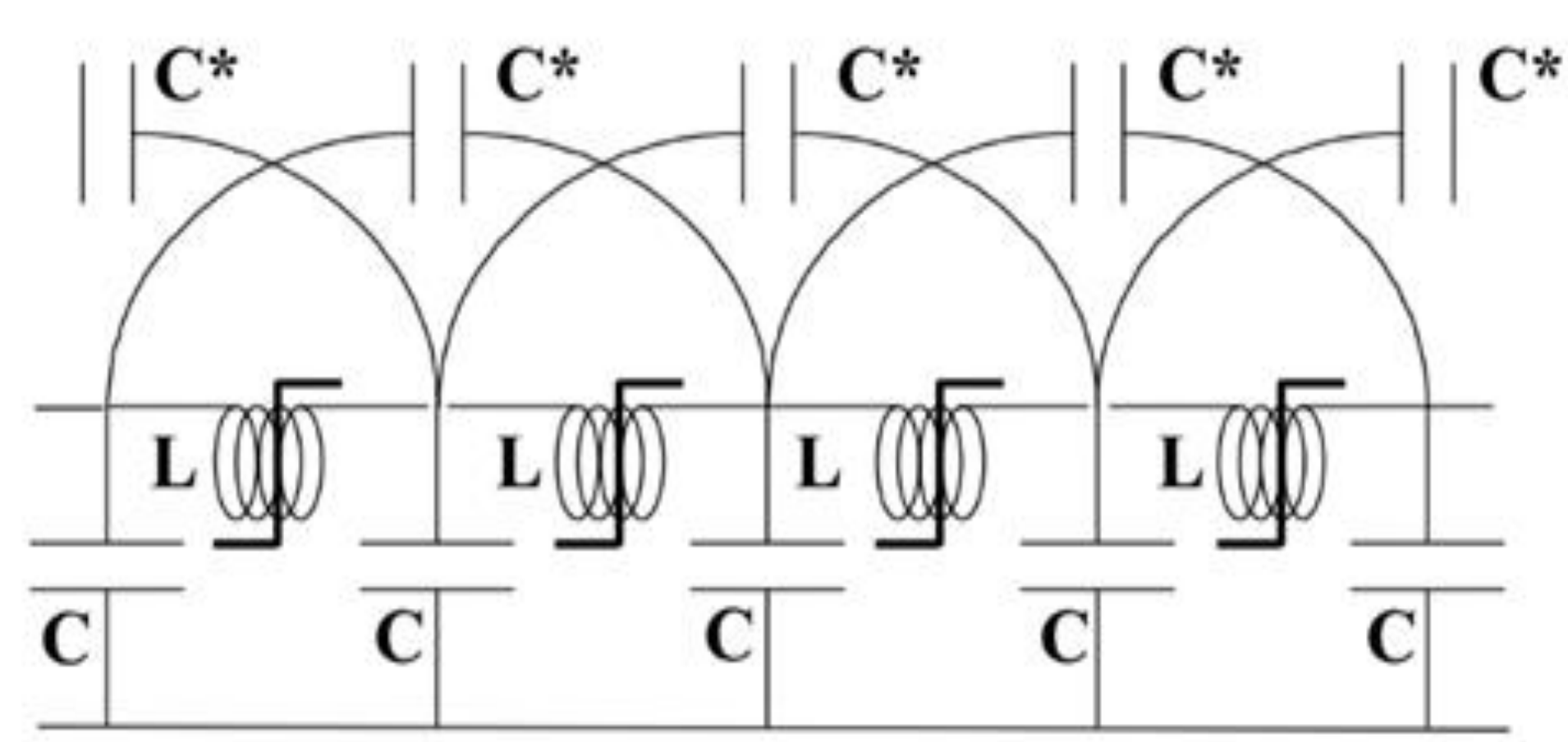

Fig. 2. Synchronous NLTL

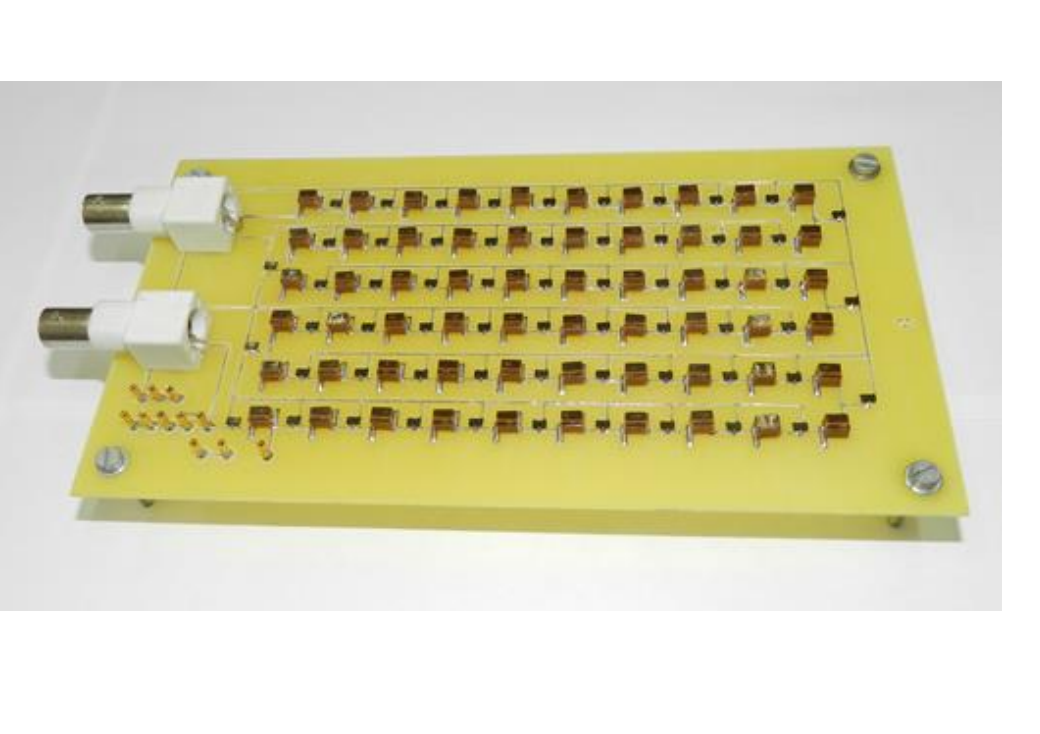

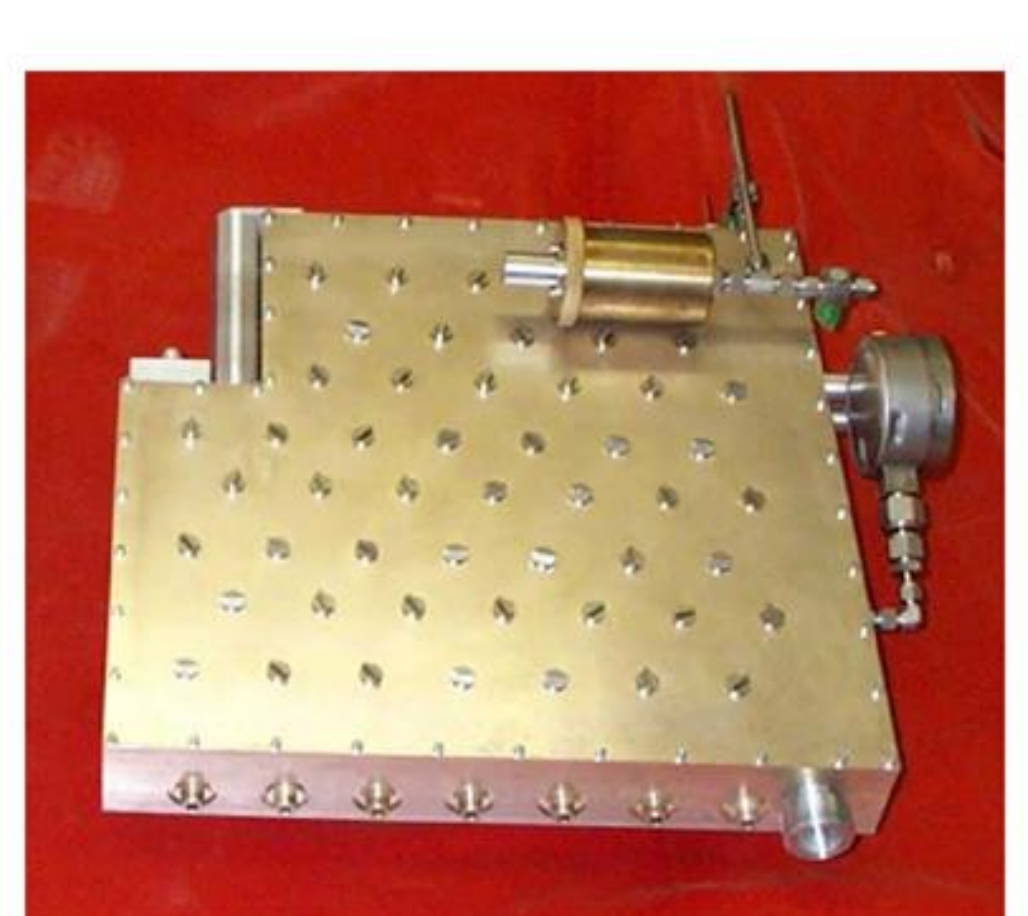

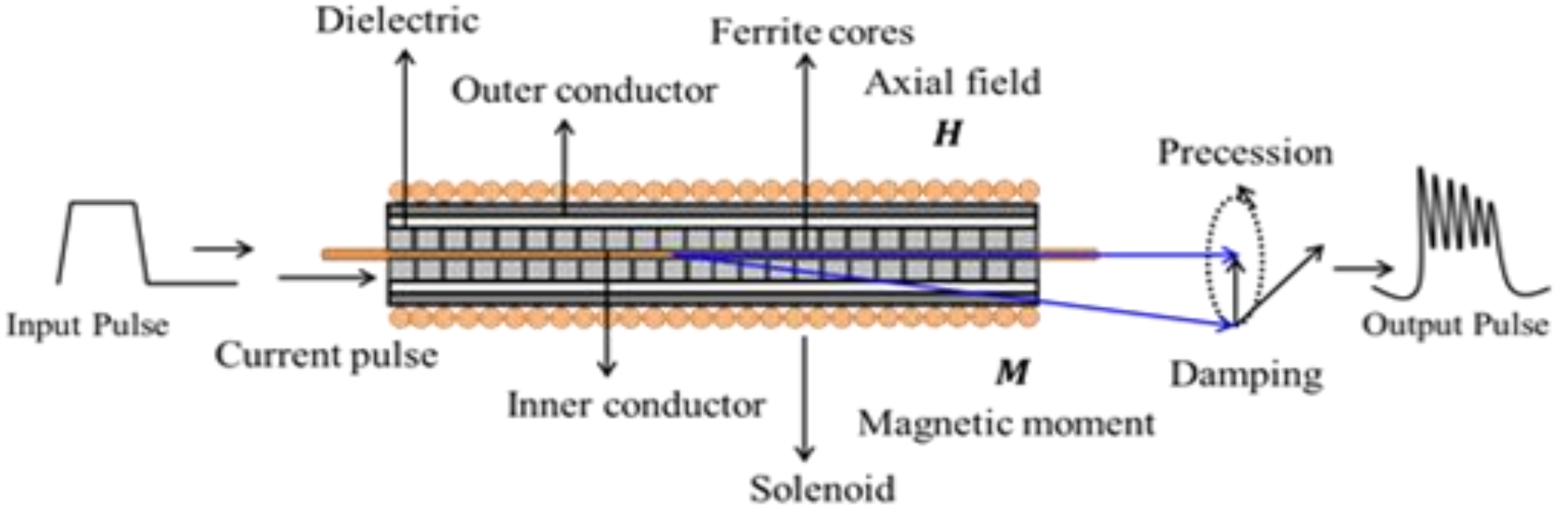

Fig. 3. Gyromagnetic NLTL

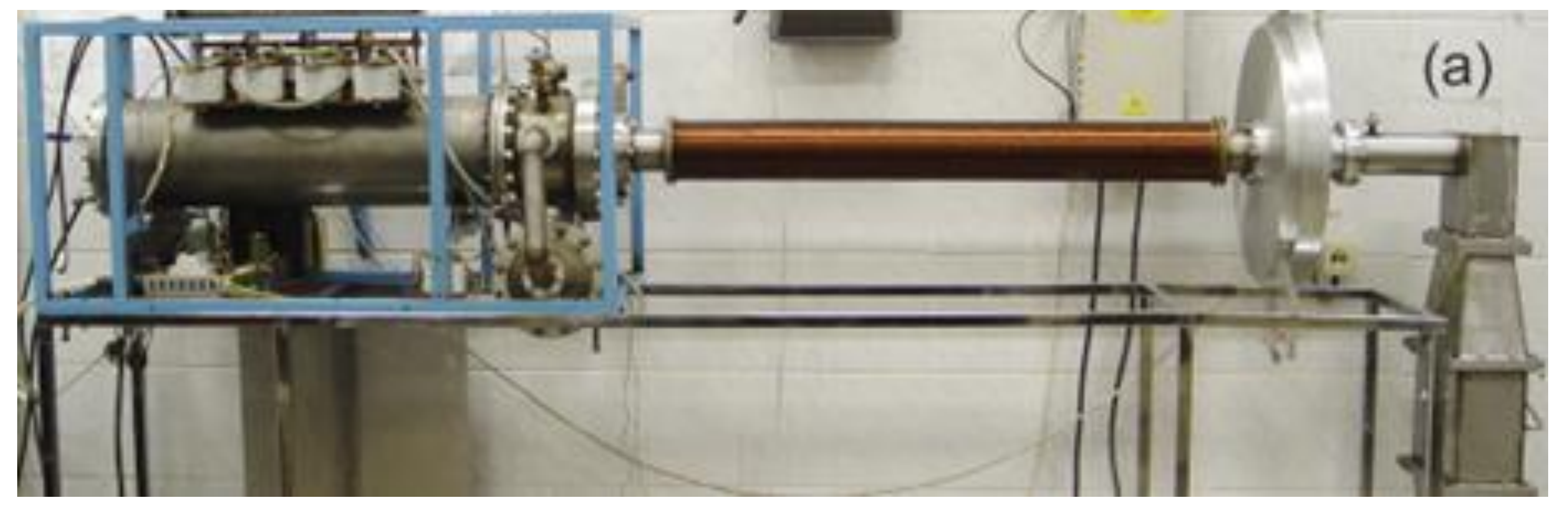

II. Relevant Experimental Results in the Literature

\begin{tabular}{|c|c|c|c|c|c|}
\hline Structure Type & Key Material & Input Pulse (kV) & Frequency Range (GHz) & Output Power & Reference \\
\hline CPW & HBV diode & 0.008 & $231-247.5$ & $10 \mathrm{~mW}$ & X. Mélique et al. - 1999 \\
\hline PCB & Varactor diode & 0.02 & $0.12-0.25$ & $98 \mathrm{~W}$ & J. Darling -2009 \\
\hline Parallel Plate & Blocks of $\mathrm{Sr}_{0.4} \mathrm{Ba}_{0.6} \mathrm{TiO}_{3}$ & 14 & $0.25-0.40$ & 15.6 MW & Ikezi et al. - 1991 \\
\hline PCB & Ceramic Capacitor & 30 & $0.01-0.09$ & $12 \mathrm{MW}$ & J. Darling -2009 \\
\hline \multirow{6}{*}{ Coaxial } & Ferrite & $30-50$ & $0.20-2.00$ & $20 \mathrm{MW}$ & N. Seddon et al. - 2007 \\
\hline & Ferrite $(\mathrm{NiZn})$ & 260 & $0.95-1.45$ & $100 \mathrm{MW}$ & D. M. French et al. - 2014 \\
\hline & Ferrite $(\mathrm{YIG})$ & $3-6$ & $1.70-1.80$ & $200 \mathrm{~kW}$ & D. V. Reale et al. - 2014 \\
\hline & Ferrite & $30-50$ & $2-6$ & $1.3 \mathrm{MW}$ & Bragg et al. - 2012 \\
\hline & Ferrite $(\mathrm{NiZn})$ & $150-270$ & $0.60-1.00$ & $3 \mathrm{GW}$ & Romanchenko et al. - 2017 \\
\hline & Ferrite $(\mathrm{NiZn})$ & 200 & $2.0-2.20$ & $200 \mathrm{MW}$ & Romanchenko et al. - 2017 \\
\hline
\end{tabular}

\section{Materials Properties versus NLTLs Performance}

PROPERTIES OF NONLINEAR DIELECTRIC MATERIALS

Nonlinearity of relative permittivity behavior with applied

voltage $(\geq 70 \%)$ with medium to low values $\left(\varepsilon_{r}<100\right)$

Low dielectric losses

(high relaxation frequency of electric dipoles)

High dielectric strength (breakdown voltage)

\section{NLTLs Performance}

Higher operation frequency

RF conversion efficiency

Power handling capability
Properties of ferrimagnetic materials

High relative permeability

Low magnetic loss

(high resistivity, low resonance linewidth)

High saturation flux

\section{NLTLs Performance}

Higher operation frequency

RF conversion efficiency

\section{Conclusions}

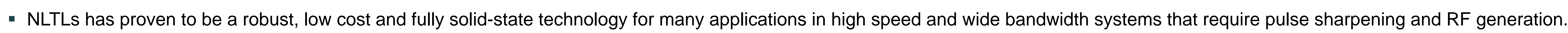

- The performance of LTNLs is related to the properties of nonlinear dielectric and ferrimagnetic materials.

- The efficiency of NLTLs is strongly affected by the energy losses in the nonlinear dielectric and ferrimagnetic materials.

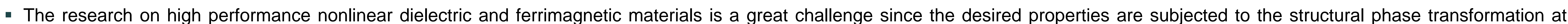
Curie temperature.

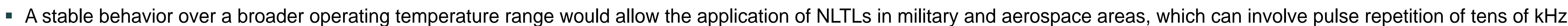

